\title{
A Mathematical Model to Examination the Behavior of Two Competing Biological Species under the Effect of a Toxicant
}

\author{
Gauri Agrawal, Alok Agrawal, Anuj Kumar Agarwal, Piyush Kumar Tripathi
}

\begin{abstract}
It is well known that the toxicants present in the environment affect the growth of any biological population living in that habitat. It also affects the carrying capacity of the environment with respect to that biological population. In this paper we are considering two logistically growing biological populations competing for a common resource under the effect of a toxicant and we've assumed that the first population discharges toxicant which is harmful to the second population only. Since, condition of the population and their habitat are limited therefore, keeping the above in the mind, here we've proposed a mathematical model to study the behaviour of the two competing population and observed that one species dies away as the time lapses due to the effect of the toxicants. It has been shown further that under certain conditions both the competing species can coexist in a long run.
\end{abstract}

Keywords : competing populations, toxicants, growth rate, carrying capacity,.

\section{INTRODUCTION}

On the Earth, the behavior of every living thing can be studied using population biology. When researchers begin to evaluate a population of species, they use many tools to help them gather information such as: observing the growth of biological population, competition for resources between biological populations in same ecological niche and so on. Since, conditions of the population and their habitat are limited, so mathematical models are used to make predictions for growth and survival of biological population in the environment. Let's look some of the population biological studies based on mathematical models: the effect of one toxicant, simultaneous effects of two or more toxicants on biological population, allelopathic effect on two competing population, effect of toxicants in case of deformity (Agarwal et al, 2016; Agrawal et al, 2000; Deluna and Hallam, 1987; Dubey et al, 2010; Dubey and Hussain, 2000; Kumar et al, 2016; Shukla and Agrawal, 1999; Shukla and Dubey, 1996; Shukla et al 2001). In 1996, Shukla and Dubey considered the concurrent impact of two toxicants for the cases of instantaneous spill, constant and periodic emission

Revised Manuscript Received on September 25, 2019

* Correspondence Author

Gauri Agrawal, Department of Mathematics, Amity University Uttar Pradesh, Lucknow, India

Alok Agrawal, Department of Mathematics, Amity University Uttar Pradesh, Lucknow, India

Anuj Kumar Agrawal, Departmet of Mathematics, BBD University, Lucknow, India

Piyush Kumar Tripathi, Department of Mathematics, Amity University Uttar Pradesh, Lucknow, India

of each of the toxicant into the environment. In 1999, Shukla and Agrawal proposed some mathematical model in the field of ecotoxicology for the impact of at least one toxicants on a single or two interacting organic species. In 2000, Dubey and Hussain proposed and analyzed a mathematical model to consider the coexistence of two contending plant species in same biological specialty. Shukla et al, 2001 considered the impact of a toxicant discharged from external sources on two contending biological species. Dubey et al 2010 studied an additional phenomenon of a chemical defense mechanism including two contending species. In this case both species emitted the toxicant in the environment and affecting the other. Further, In 2016, Agarwal et al and Kumar et al considered the effect of a solitary toxicant when toxicant discharged by organic species itself and discharged by some outer sources respectively. In these studies, they show that after-effect of toxicant some members loose their reproduction capability.

In population biology, there may exist some conditions where two biological populations living in same ecological niche make a competition for resources. Out of these two populations, one population produces a toxicant which affects the growth rate of other biological population. It also affects the carrying capacity of the environment regarding the second population.

For example, plants growing in the close proximity to black walnuts often develop mysterious illnesses without warning. Leaves on annuals yellow, wilt and die due to the allelopathic effect that the chemical juglone, produced by walnuts, has on vegetable plants like cabbage, peppers, tomatoes, potatoes etc. Allelopathy refers to the harmful effects of one plant on another plant, from the release of biochemicals, known as allelochemicals to inhibit germination or impede development of nearby plant life.

We consider two logistically growing biological species living in a habitat and competing for common resource. Let $\mathrm{N} \_1(\mathrm{t})$ and $\mathrm{N} \_2(\mathrm{t})$ be the population densities of the two biological species at time $\mathrm{t} . \mathrm{T}(\mathrm{t})$ is the environmental concentration of the amount being emitted by the first population (with population density $N_{-} 1(T)$. U(T) is the uptaken amount of toxicant by the second population. This uptaken toxicant increases the mortality rate in second population. The amount of toxicant present in the environment decreases the carrying capacity of the environment with respect to the 2 nd population. We assume that this toxicant is not harmful to the 1 st population. 
Keeping the above in the mind, we propose the following mathematical model.

$$
\begin{gathered}
\text { II. MATHEMATICAL MODEL } \\
\frac{d N_{1}}{d t}=r_{1} N_{1}-r_{1} \frac{N_{1}^{2}}{K_{1}}-\theta_{1} N_{1} N_{2} \ldots \ldots \ldots \text { (1) } \\
\frac{d N_{2}}{d t}=r_{2} N_{2}-r_{2} \frac{N_{2}^{2}}{K_{2}(T)}-d N_{2} U-\theta_{2} N_{1} N_{2} \ldots \ldots \ldots \\
\frac{d T}{d t}=\lambda N_{1}-\delta T-\gamma T N_{2}+\pi v N_{2} U \ldots \ldots \ldots \text { (3) } \\
\frac{d U}{d t}=\gamma T N_{2}-\beta U-v N_{2} U \ldots \ldots \ldots(4)
\end{gathered}
$$

Here the growth rates of the populations are $\mathrm{r}_{-} 1, \mathrm{r} \_2 . \mathrm{K} \_1$ and $\mathrm{K} \_2$ represent the carrying capacities of the environment with respect to the populations. $\theta \_1, \theta \_2$ are the competition coefficients. $d$ is the mortality rate of the second species due to uptake of toxicant. $\lambda$ is the rate by which first species is producing toxicant. The natural wash out rate of $\mathrm{T}$ is $\delta>0$. $\gamma>0$ depletion rate coefficient of $\mathrm{T}(\mathrm{t})$ due to its uptake by members of second species . $\beta>0$ is the natural wash out rate coefficient of $U(t) . v>0$ is the depletion rate coefficient of $U(t)$ due to decay of some members of $\mathrm{N} \_2$ ( $\mathrm{t}$ ) and a fraction $\pi(0 \leq \pi \leq 1)$ of which may re-enter into the environment.

$$
K_{2}(0)=K_{20}>0, \frac{d K_{2}}{d T}<0 \text { for } T>0
$$

\section{EQUILIBRIUM POINTS}

The model given above has four equilibrium points namely

$E_{1}(0,0,0,0), E_{2}\left(0, K_{20}, 0,0\right), E_{3}\left(K_{1}, 0, \frac{\lambda K_{1}}{\sigma}, 0\right)$

and

$E_{4}\left(N_{1}^{*}, N_{2}^{*}, T^{*}, U^{*}\right)$.

The existence of $E_{1}, E_{2}, E_{3}$ is obvious. In the following, we are giving a short proof for the existence and uniqueness of $\mathrm{E}_{4}$.

$$
\begin{gathered}
N_{1}=K_{1}\left(1-\frac{\theta_{1} N_{2}}{r_{1}}\right)=h_{1}\left(N_{2}\right) \quad \text { (say) } \cdots \cdots \cdots \text { (5) } \\
N_{2}=K_{2}(T)\left[1-\frac{d U+\theta_{2} N_{1}}{r_{2}}\right]=h_{2}\left(N_{2}\right) \text { (say) } \cdots \cdots \cdots(\text { (6) } \\
T=\frac{\lambda K_{1}\left(r_{1}-\theta_{1} N_{2}\right)+r_{1} \pi v N_{2} U}{r_{1}\left(\delta+\gamma N_{2}\right)}=g\left(N_{2}\right) \quad \text { (say) } \cdots \cdots \cdots \text { (7) } \\
\text { and } U=\frac{\gamma \lambda K_{1}\left(r_{1}-\theta_{1} N_{2}\right) N_{2}}{r_{1} f\left(N_{2}\right)}=h\left(N_{2}\right) \quad \text { (say) } \cdots \cdots \cdots \text { (8) }
\end{gathered}
$$

Where

$$
f\left(N_{2}\right)=\beta \delta+(\beta Y+v \delta) N_{2}+(1-\pi) \gamma v N_{2}^{2}
$$

Then we can see that $f\left(\mathrm{~N}_{2}\right)>0$ for $\mathrm{N}_{2}>0$
Now, it can be verified that,

$$
h_{1}\left(N_{2}\right)>0, h_{2}\left(N_{2}\right)>0 \text { and } \frac{d h_{1}}{d N_{2}}=-\frac{\theta_{1} R_{1}}{r_{1}}<0
$$

Let

$$
\begin{aligned}
& F\left(N_{2}\right)=r_{2} N_{2}-K_{2}\left(h\left(N_{2}\right)\right)\left[r_{2}-d \cdot g\left(N_{2}\right)-\theta_{2} \cdot h_{1}\left(N_{2}\right)\right] \\
& -(9) \\
& \text { then } F(0)=0-K_{20}\left[r_{2}-d_{0} 0-\theta_{2} K_{1}\right] \\
& =-K_{20}\left[r_{2}-\theta_{2} K_{1}\right] \\
& <0 \text {, provided } r_{2}-\theta_{2} K_{1}>0
\end{aligned}
$$

And $F\left(K_{20}\right)>0$, provided $r_{1}-\theta_{1} K_{20}>0$.

It can be seen that $F^{v}\left(N_{2}\right)>0$ in the interval $\left(0, K_{20}\right)$

This shows that $F\left(\mathrm{~N}_{2}\right)=0$ has a unique root say $\mathrm{N}_{2}^{*}$ in $\left(0, K_{20}\right)$. Once $N_{2}^{*}$ is found, $N_{1}^{*}, T^{*} \& U^{*}$ can be found using equations (5), (7), (8). Hence, existence and uniqueness of $E_{4}$ is established.

\section{LOCAL STABILITY ANALYSIS}

We construct the variational matrix for finding the Local stability behaviour of the equilibrium points.

$M=\left(\begin{array}{cccc}r_{1}-\frac{2 r_{1} N_{1}}{K_{1}}-\theta_{1} K_{20} & -\theta_{1} N_{1} & 0 & 0 \\ -\theta_{2} N_{2} & r_{2}-\frac{2 r_{2} N_{2}}{K_{2}}-d U-\theta_{2} N_{2} & 0 & -d N_{2} \\ \lambda & -\gamma T+\pi v U & -\delta-\gamma N_{2} & \pi v N_{2} \\ 0 & \gamma T-v U & \gamma N_{2} & -\beta-v N_{2}\end{array}\right)$

At $E_{1}(0,0,0,0)$.

$$
M_{1}=\left(\begin{array}{cccc}
r_{1} & 0 & 0 & 0 \\
0 & r_{2} & 0 & 0 \\
\lambda & 0 & -\delta & 0 \\
0 & 0 & 0 & -\beta
\end{array}\right)
$$

It is clear that the first two eigen values are +ve whereas the last two eigen values are-ve, which shows that $E_{1}$ is a saddle point unstable in $N_{1}-N_{2}$ plane and stable in $T-U$ plane. At $E_{2}\left(0, K_{20}, 0,0\right)$

$M_{2}=\left(\begin{array}{cccc}r_{1}-\theta_{1} K_{20} & 0 & 0 & 0 \\ -\theta_{2} K_{20} & -r_{2} & 0 & -d K_{20} \\ \lambda & 0 & -\delta-\gamma K_{20} & \pi v K_{20} \\ 0 & 0 & \gamma K_{20} & -\beta-v K_{20}\end{array}\right)$

$\left|M_{2}-x I\right|=0 \Rightarrow$

$$
\begin{aligned}
& \left|\begin{array}{cccc}
r_{1}-\theta_{1} K_{20}-x & 0 & 0 & 0 \\
-\theta_{2} K_{20} & -r_{2}-x & 0 & -d K_{20} \\
\lambda & 0 & -\delta-\gamma K_{20}-x & \pi v K_{20} \\
0 & 0 & \gamma K_{20} & -\beta-v K_{20}-x
\end{array}\right|=0 \\
& \Rightarrow\left(r_{1}-\theta_{1} K_{20}-x\right)\left|\begin{array}{ccc}
-r_{2}-x & 0 & -d K_{20} \\
0 & -\delta-\gamma K_{20}-x & \pi v K_{20} \\
0 & \gamma K_{20} & -\beta-v K_{20}
\end{array}\right|=0
\end{aligned}
$$

$$
\Rightarrow\left(r_{1}-\theta_{1} K_{20}-x\right)\left(-r_{2}-x\right)\left|\begin{array}{cc}
-\delta-\gamma K_{20}-x & \pi v K_{20} \\
\gamma K_{20} & -\beta-v K_{20}-x
\end{array}\right|=0
$$

$$
\Rightarrow\left(r_{1}-\theta_{1} K_{20}-x\right)\left(-r_{2}-x\right)\left\{\left(-\beta-v K_{20}-x\right)\left(-\delta-\gamma K_{28}-v\right)-\pi v y K_{20}^{2}\right\}=0
$$


Now for the other two eigen values, the expression in the curly bracket gives

$$
\left(\beta+v K_{20}+x\right)\left(\delta+\gamma K_{20}+x\right)+\pi v \gamma K_{20}^{2}=0
$$

$\Rightarrow x^{2}+\left(\beta+v K_{20}+\delta+\gamma K_{20}\right) x+\left(\beta+v K_{20}\right)\left(\delta+\gamma K_{20}\right)+\pi v \gamma K_{20}^{2}=0$

$\Rightarrow x^{2}+b x+c=0$

$\Rightarrow x=\frac{-b \pm \sqrt{b^{2}-4 c}}{2}$

$\Rightarrow b^{2}-4 c \leq b^{2}$

or $4 c \geq 0$ or $c \geq 0$, which is satisfied.

Therefore, if $r_{1}<\theta_{1} K_{20}$ then all the eigen values are either -ve or having -ve real parts .

$\Rightarrow E_{2}$ is Locally Asymptotically Stable.

At $E_{a}\left(K_{1}, 0, \frac{\lambda K_{1}}{a}, 0\right)$

$$
M_{a}=\left(\begin{array}{cccc}
-r_{1} & -\theta_{1} K_{1} & 0 & 0 \\
0 & r_{2}-\theta_{2} K_{1} & 0 & 0 \\
\lambda & \frac{-\gamma \lambda K_{1}}{\delta} & -\delta & 0 \\
0 & \frac{\gamma \lambda K_{1}}{\delta} & 0 & -\beta
\end{array}\right)
$$

$\left|M_{a}-x I\right|=0$

$$
\begin{aligned}
& \Rightarrow\left|\begin{array}{cccc}
-r_{1}-x & -\theta_{1} K_{1} & 0 & 0 \\
0 & r_{2}-\theta_{2} K_{1}-x & 0 & 0 \\
\lambda & \frac{-\gamma \lambda K_{1}}{\delta} & -\delta-x & 0 \\
0 & \frac{\gamma \lambda K_{1}}{\delta} & 0 & -\beta-x
\end{array}\right|=0 \\
& \Rightarrow(-\beta-x)\left|\begin{array}{ccc}
-r_{1}-x & -\theta_{1} K_{1} & 0 \\
0 & \frac{r_{2}-\theta_{2} K_{1}-x}{2} & 0 \\
\lambda & \frac{-\gamma \lambda K_{1}}{\delta} & -\delta-x
\end{array}\right|=0 \\
& \Rightarrow(-\beta-x)(-\delta-x)\left|\begin{array}{cc}
-r_{1}-x & -\theta_{1} K_{1} \\
0 & r_{2}-\theta_{2} K_{1}-x
\end{array}\right|=0 \\
& \Rightarrow(\beta+x)(\delta+x)\left(r_{1}+x\right)\left(r_{2}-\theta_{2} K_{1}-x\right)=0
\end{aligned}
$$

If $r_{2}-\theta_{2} K_{1}<0 \Rightarrow \mathrm{E}_{a}$ is Locally Asymptotically Stable.

$$
\text { At } E_{4}\left(N_{1}^{*}, N_{2}^{*}, T^{*}, U^{*}\right) \text {, }
$$

$M_{4}=$

$$
=\left(\begin{array}{cccc}
-\frac{r_{1} N_{1}^{*}}{K_{1}} & -\theta_{1} N_{1}^{*} & 0 & 0 \\
-\theta_{2} N_{2}^{*} & -\frac{r_{2} N_{2}^{*}}{K_{2}\left(T^{*}\right)} & 0 & -d N_{2}^{*} \\
\lambda & -\left(\gamma T^{*}-\pi v U^{*}\right) & -\left(\delta+\gamma N_{2}^{*}\right) & \pi v N_{2}^{*} \\
0 & \gamma T^{*}-v U^{*} & \gamma N_{2}^{*} & -\left(\beta+v N_{2}^{*}\right)
\end{array}\right)
$$

$\left|M_{4}-x I\right|=0$

$$
\begin{aligned}
\Rightarrow \quad\left(-r_{1} \frac{N_{1}^{*}}{K_{1}}-x\right)\left|\begin{array}{ccc}
-\frac{r_{2} N_{2}^{*}}{K_{2}\left(T^{*}\right)}-x & 0 & -d N_{2}^{*} \\
-\left(\gamma T^{*}-\pi v U^{*}\right. & -\left(\delta+\gamma N_{2}^{*}\right)-x & \pi v N_{2}^{*} \\
\gamma T^{*}-v U^{*} & \gamma N_{2}^{*} & -\left(\beta+v N_{2}^{*}\right)-x
\end{array}\right| \\
+\theta_{1} N_{1}^{*}\left|\begin{array}{ccc}
-\theta_{2} N_{2}^{*} & 0 & -d N_{2}^{*} \\
\lambda & -\left(\delta+\gamma N_{2}^{*}\right)-x & \pi v N_{2}^{*} \\
0 & \gamma N_{2}^{*} & -\left(\beta+v N_{2}^{*}\right)-x
\end{array}\right|=0
\end{aligned}
$$

Or

$$
\left(\frac{r_{1} N_{1}^{*}}{R_{1}}+x\right)\left[( \frac { r _ { 2 } N _ { 2 } ^ { * } } { R _ { 2 } ( T ^ { * } ) } + x ) \left\{\left(\delta+\gamma N_{2}^{*}+x\right)\left(\beta+v N_{2}^{*}+x\right)-\right.\right.
$$
$\left.\pi v \gamma N_{2}^{* 2}\right\}+d N_{2}^{*}\left\{-\left(\gamma T^{*}-\pi v U^{*}\right) \gamma N_{2}^{*}+\left(\gamma T^{*}-v U^{*}\right)(\delta+\right.$ $\left.\left.\left.v N_{2}^{*}+x\right)\right\}\right]+\theta_{1} N_{1}^{*}\left[-\theta_{2} N_{2}^{*}\left\{\left(\delta+\gamma N_{2}^{*}+x\right)\left(\beta+v N_{2}^{*}+\right.\right.\right.$ $\left.\left.x)-\pi v \gamma N_{2}^{* 2}\right\}-d \lambda y N_{2}^{* 2}\right]=0$

or $\left(\frac{p_{1} N_{1}^{*}}{R_{1}}+x\right)\left[\left(\frac{F_{2} N_{2}^{*}}{W_{2}\left(T^{*}\right)}+x\right)\left\{x^{2}+\left(\delta+\gamma N_{2}^{*}+\beta+\nu N_{2}^{*}\right) x+\beta \delta+\delta N N_{2}^{*}+\beta \gamma N_{2}^{*}+(1-\pi) w N_{2}^{*}\right\}\right.$

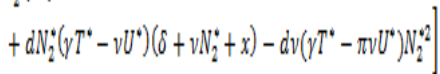
$-\theta_{1} \theta_{2} N_{1}^{*} N_{2}^{*}\left\{x^{2}+\left(\delta+\gamma N_{2}^{*}+\beta+v N_{2}^{*}\right) x+\beta \delta+\delta v N_{2}^{*}+\beta \gamma N_{2}^{*}+(1-\pi) v N_{2}^{2}\right\}-d \lambda \gamma N_{2}^{2}=0$

or $\left(\frac{r_{1} N_{1}^{*}}{R_{1}}+x\right)\left[\left(\frac{r_{2} N_{2}^{*}}{\mathbb{R}_{2}\left(T^{*}\right)}+x\right)\left\{x^{2}+\left(\delta+\gamma N_{2}^{*}+\beta+v N_{2}^{*}\right) x+\beta \delta+\delta v N_{2}^{*}+\beta \gamma N_{2}^{*}+(1-\pi) w N_{2}^{*}\right\}+d N_{2}^{*}\left(\gamma T^{*}-\nu W^{*}\right) x\right.$ $\left.+d N_{2}^{*}\left(\delta \gamma T^{*}+\gamma^{2} N_{2}^{*} T^{*}-\delta V V^{*}-w N N_{2}^{*} V^{*}\right)-d \gamma^{2} T^{*} N_{2}^{*}-(1-\pi) d v V^{*} N_{2}^{*}\right]$

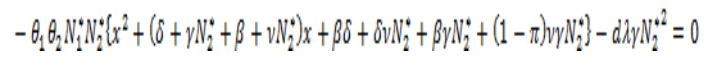
or $\left(\frac{r_{1} N_{1}^{*}}{K_{1}}+x\right)\left[\left(\frac{r_{2} N_{2}^{*}}{V_{2}\left(T^{*}\right)}+x\right)\left(x^{2}+a_{1} x+a_{2}\right)+\beta d U^{*} x+\beta \delta d U^{*}-(1-\pi) d w \gamma U^{*} N_{2}^{2}\right]-\theta_{1} \theta_{2} N_{1}^{*} N_{2}^{*}\left(x^{2}+a_{1} x+a_{2}\right)$
$-d \lambda y N_{2}^{* 2}=0$ where $a_{1}=\delta+\beta+(\gamma+v) N_{2}^{*}, a_{2}=\beta \delta+(\delta v+\beta \gamma) N_{2}^{*}+$ $(1-\pi) v \gamma N_{2}^{*}$

$$
\begin{aligned}
& \text { or }\left(\frac{r_{1} N_{1}^{*}}{R_{1}}+x\right)\left[x^{2}+\left(\frac{r_{2} N_{2}^{*}}{K_{2}\left(T^{*}\right)}+a_{1}\right) x^{2}+\left(\frac{a_{1} r_{2} N_{2}^{*}}{K_{2}\left(T^{*}\right)}+a_{2}+\beta d U^{*}\right) x+\frac{a_{2} r_{2} N_{2}^{*}}{K_{2}\left(T^{*}\right)}+\beta \delta d U^{*}-(1-\pi) d w \gamma U^{*} N_{2}^{n}\right] \\
& -\theta_{1} \theta_{2} N_{1}^{*} N_{2}^{*} x^{2}-a_{1} \theta_{1} \theta_{2} N_{1}^{*} N_{2}^{*} x+a_{2} \theta_{1} \theta_{2} N_{1}^{*} N_{2}^{*}-d \lambda y N_{2}^{2}=0 \\
& x^{4}+\left(\frac{r_{1} N_{1}^{*}}{R_{1}}+\frac{r_{2} N_{2}^{*}}{R_{2}\left(T^{*}\right)}+a_{1}\right) x^{2}+\left(\left\{\frac{r_{1} N_{1}^{*}}{R_{1}}\right)\left(\frac{r_{2} N_{2}^{*}}{R_{2}\left(T^{*}\right)}+a_{1}\right)+\frac{a_{1} P_{2} N_{2}^{*}}{R_{2}\left(T^{*}\right)}+a_{2}+\beta d W^{*}-\theta_{1} \theta_{2} N_{1}^{*} N_{2}^{*}\right) x^{2}
\end{aligned}
$$

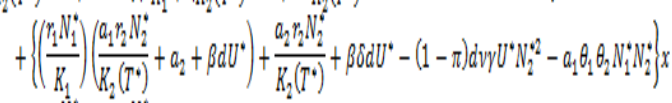

$$
\begin{aligned}
& +\left[\left(\frac{r_{1} N_{1}^{*}}{R_{1}}\right)\left(\frac{G_{2} r_{2} N_{2}^{*}}{R_{2}\left(T^{*}\right)}+\beta \delta d U^{*}-(1-\pi) d w V^{*} N_{2}^{2}\right)+a_{2} \theta_{1} \theta_{2} N_{1}^{*} N_{2}^{*}-d \lambda \gamma N_{2}^{n}\right]
\end{aligned}
$$$$
=0
$$ 
This can be written as,

$$
\Rightarrow x^{4}+b_{1} x^{a}+b_{2} x^{2}+b_{3} x+b_{4}=0
$$

Where

$$
\begin{aligned}
& b_{1}=\frac{r_{1} N_{1}^{*}}{K_{1}}+\frac{r_{2} N_{2}^{*}}{K_{2}\left(T^{*}\right)}+a_{1}, \\
& b_{2}=\left(\frac{r_{1} N_{1}^{*}}{K_{1}}\right)\left(\frac{r_{2} N_{2}^{*}}{K_{2}\left(T^{*}\right)}+a_{1}\right)+\frac{a_{1} r_{2} N_{2}^{*}}{K_{2}\left(T^{*}\right)}+a_{2}+\beta d U^{*}-
\end{aligned}
$$$$
\theta_{1} \theta_{2} N_{1}^{*} N_{2}^{*}
$$

$$
\begin{aligned}
& b_{1}=\left(\frac{r_{1} N_{1}^{*}}{K_{1}}\right)\left(\frac{a_{1} r_{2} N_{2}^{*}}{R_{2}\left(T^{*}\right)}+a_{2}+\beta d U^{*}\right)+\frac{a_{2} r_{2} N_{2}^{*}}{R_{2}\left(T^{*}\right)}+\beta \delta d U^{*}-(1-\pi) d v U^{*} N_{2}^{n}-a_{1} \theta_{1} \theta_{2} N_{1}^{*} N_{2}^{*} \\
& b_{4}=\left(\frac{r_{1} N_{1}^{*}}{K_{1}}\right)\left(\frac{a_{2} r_{2} N_{2}^{*}}{K_{2}\left(T^{*}\right)}+\beta \delta d U^{*}-(1-\pi) d v U^{*} N_{2}^{*}\right)+a_{2} \theta_{1} \theta_{2} N_{1}^{*} N_{2}^{*}-d \lambda \gamma N_{2}^{* 2}
\end{aligned}
$$
are either -ve or with - ve real parts provided,

$$
\left.\begin{array}{l}
\text { (i) } b_{1}, b_{2}, b_{a}, b_{4}>0 \\
\text { (ii) } b_{1}, b_{2}>b_{a} \\
\text { (iii) } b_{1} b_{2} b_{a}>b_{a}^{2}+b_{1}^{2} b_{4}
\end{array}\right\}
$$

It can be seen that $b_{1}>0, b_{2}$ is +ve provided $r_{2}-\theta_{2} K_{1}>0$ and $r_{1}-\theta_{1} K_{20}>0$. Since both of these conditions are used for establishing the existence and

Thus $\mathrm{E}_{4}$ is Locally Asymptotically Stable provided condition

\section{NUMERICAL SIMULATION}

To give a better prediction for the case when two competing biological species living in same environment, we present here numerical simulation of mathematical model (1-4) by assuming the carrying capacity function $K_{2}(T)$ corresponding to the second population $\mathrm{N}_{2}$ as:

$$
K_{2}(T)=K-\frac{b_{1} T}{1+b_{2} T}
$$

and set of parameters

$$
\begin{aligned}
& r_{1}=0.55, \quad d=0.006, \quad K=10.0, \quad K_{1}=10.0, \quad b_{1}=0.02, \quad b_{2}=1, \\
& r_{2}=0.8, \quad \theta_{1}=0.01, \quad \theta_{2}=0.02, \quad \lambda=0.05, \quad \delta=0.08, \quad \gamma=0.005,
\end{aligned}
$$

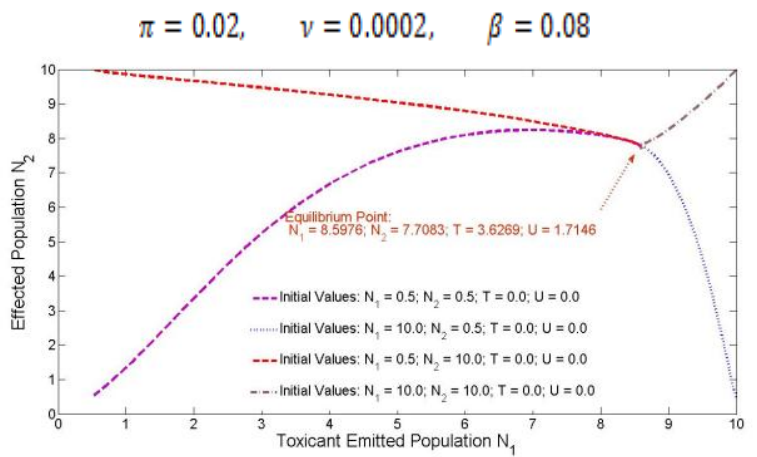

Figure 1 : Model system become stable at the equilibrium point
By Routh - Hurwitz's Criterion , the roots of the polynomial uniqueness of $E_{4}$, so these are obviously true. (14) are satisfied.

For the above set of parameters the model system (1-4) becomes stable at the equilibrium point $\left(N_{1}=8.5976 ; N_{2}=7.7083 ; T=3.6269 ; U=1.7146\right)$ for the different initial values.

In Figure $2 \& 3$, we have shown the effect of toxicant emission rate $\lambda$ on both biological population (toxicant emitting population $N_{1}$ and toxicant effected population $N_{2}$ respectively). Figure 3 shows that as the toxicant emission rate increases, the density of toxicant effected biological population decreases. Since toxicant emitting population $N_{1}$ also make competition for resources with toxicant effected population $N_{2}$, therefore as the toxicant effected population decreases, toxicant emitting population $N_{1}$ increases due to increase of emission rate of toxicant (see Figure 2).

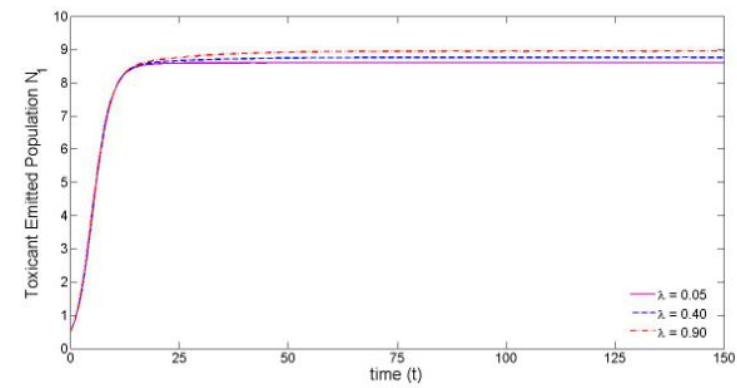

Figure 2: Variation in toxicant emitted population $N_{-} 1$ corresponding to the toxicant emission rate $\lambda$

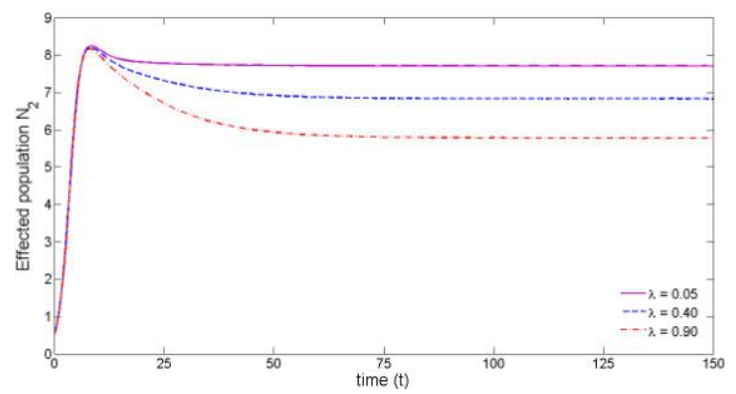

Figure 3: Variation in toxicant effected populationN_2 corresponding to the toxicant emission rate $\lambda$

In Figure $4 \& 5$, we have shown the effect of mortality rate $d$ (due to uptake of the toxicant by the toxicant effected population) on both biological population (toxicant emitting population $N_{1}$ and toxicant effected population $N_{2}$ respectively). Same perception in the aboveparagraph, the toxicant emitting population $N_{1}$ increases and toxicant effected population decreases with increasing $d$.

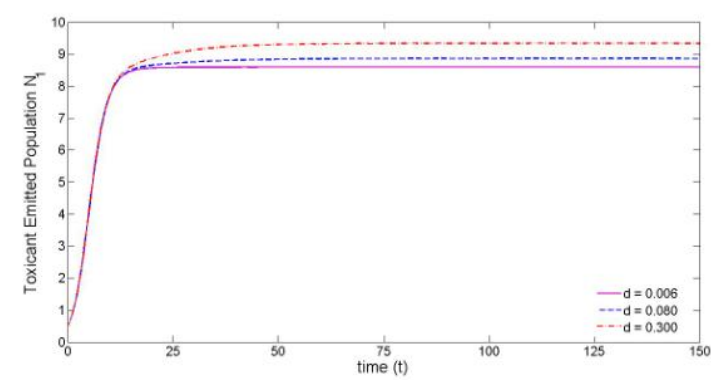

Figure 4: Variation in toxicant effected population $\mathrm{N}_{-} 1$ corresponding to $\mathrm{d}$.

Published By

Blue Eyes Intelligence Engineering 


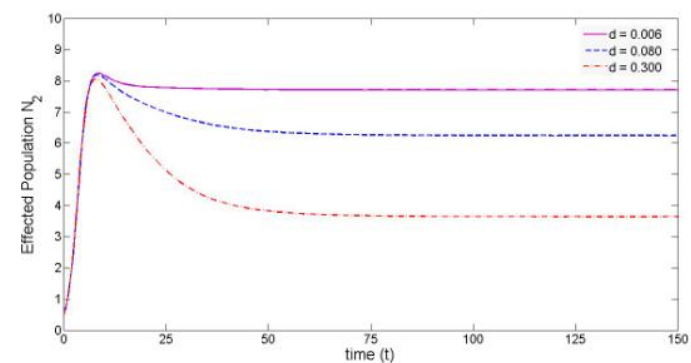

Figure 5: Variation in toxicant effected population N_2 corresponding to $\mathrm{d}$

\section{CONCLUSION}

In this work, we have considered a situation, where two biological species are competing for common resource under the effect of a toxicant. The toxicant is assumed to be produced by the first species and it is assumed to be harmful to the second species only. The model has four equilibrium pointsnamely

$E_{1}(0,0,0,0), E_{2}\left(0, K_{20}, 0,0\right), E_{a}\left(K_{1}, 0, \frac{\lambda K_{1}}{g}, 0\right) \quad$ and $E_{4}\left(N_{1}^{*}, N_{2}^{*}, T^{*}, U^{*}\right)$.The zero equilibrium $E_{1}$ is shown to be a saddle point whereas two non negative equilibrium points $E_{2}$ and $E_{a}$ are shown to be conditionally stable. In either of these two cases, one species dies away as the time lapses. The fourth equilibrium $E_{4}$ which is the interior equilibrium is shown to be stable under certain conditions. This means that both the competing species can coexist in a long run.

\section{REFERENCES}

1. Agarwal, A. K., Khan, A. W., \&Agrawal, A. K. (2016). The effect of an external toxicant on a biological species in case of deformity: a model. Modeling Earth Systems and Environment, 2(3), 148.

2. Agrawal, A. K., Dubey, B., Sinha, P., \&Shukla, J. B. (2000). Effects of two or more toxicants on a biological species: A non linear mathematical model and its analysis. Mathematical Analysis and Applications, A. P Dwivedi (editor), Narosa Publishing House, New Delhi, 93-109.

3. Deluna, J. T. \& Hallam. T. G. (1987): Effect of toxicants on population: a qualitative approach IV: Resource-consumer-toxicant models. Ecol Model., 35, 249-273.

4. Dubey, B. \& Hussain, J. (2000): A model for the allelopathic effect on two competing species. Ecologicl Modelling, 129(2-3), 195-207.

5. Dubey, B., Shukla, J. B., Sharma, S., Agarwal, A. K., \& Sinha, P. (2010): A mathematical model for chemical defense mechanism of two competing species. Nonlinear Analysis: Real World Applications, 11(2), 1143-1158.

6. Kumar, A., Agrawal, A. K., Hasan, A. \& Misra, A. K. (2016): Modeling the effect of toxicant on the deformity in a subclass of a biological species. Modeling Earth Systems and Environment, 2(1), 40.

7. La Salle, J. \& Lefschetz, S. (2012): Stability by Liapunov's Direct Method with Applications by Joseph L Salle and Solomon Lefschetz (Vol. 4). Elsevier.

8. Shukla, J. B. \& Agrawal, A. K. (1999): Some mathematical models in ecotoxicology: Effects of toxicants on biological species. Sadhana, 24(1-2), 25-40.

9. Shukla, J. B., Agrawal, A. K., Dubey, B. \& Sinha, P. (2001): Existence and survival of two competing species in a polluted environment: a mathematical model. Journal of Biological Systems, 9(2), 89-103.

10. Shukla, J. B. \& Dubey, B. (1996): Simultaneous effect of two toxicants on biological species: a mathematical model. Journal of Biological Systems, 4(1), 109-130. 